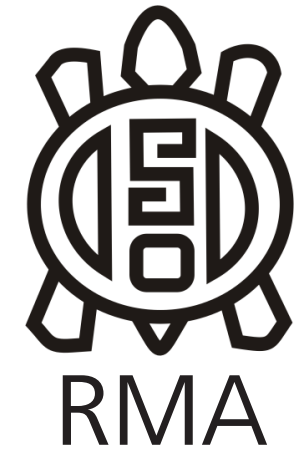

Dossier

\title{
Las piedras al fuego. Experimentos para abordar la distribución espacial de restos líticos termoalterados
}

\author{
Stones in the fire. Experimental studies on the spatial distribution \\ of thermally altered lithics \\ Ariel D. Frank* y Juan Baridón** \\ *CONICET, División Arqueología, Facultad de Ciencias Naturales y Museo, Universidad \\ Nacional de La Plata, Argentina. Email: frank.ariel@gmail.com \\ **División Arqueología, Facultad de Ciencias Naturales y Museo, Universidad Nacional \\ de La Plata, Argentina. Email: baridonjuan@gmail.com
}

\begin{abstract}
Resumen
En este trabajo, evaluamos el modo en que se distribuyen los restos líticos descartados en estructuras de combustión. Realizamos experimentaciones en las cuales arrojamos al fuego lascas de diversos tamaños. Una vez apagados los fogones, analizamos el modo en que quedaron dispersos los fragmentos generados. Las materias primas empleadas son variedades silíceas procedentes de la Meseta Central de Santa Cruz y vidrio industrial. Los resultados indican que las distintas materias primas responden de manera diversa al calor pero también que existen tendencias vinculadas al tamaño de los artefactos. Las piezas pequeñas tienen un bajo índice de fragmentación y tienden a mantenerse dentro de la estructura de combustión. Los elementos de mayor tamaño suelen fragmentarse intensamente y pueden despedir gran cantidad de partes fuera del fogón, llegando incluso a más de 3 metros de distancia. A partir de los resultados se proponen expectativas para distintos contextos de caída de piezas al fuego.
\end{abstract}

Palabras clave: Termoalteración; Descarte; Fogones; Distribución; Experimentación.

\begin{abstract}
In this paper we analyze the way in which lithic remains discarded in hearths are distributed. We perform experimentations in which we throw flakes of different sizes into the fire. Once extinguished, we analyzed the dispersal of the fragments generated by the fire. The raw materials are industrial glass and siliceous varieties which come from the Central Plateau of Santa Cruz. Results show that each raw material responds to heat in a different way. However, there are also some tendencies related to the size of the artifacts. Small flakes don't break much and stay within the hearth. Big flakes, on the other hand, usually break intensely and can expel a great amount of fragments outside the hearth, reaching more than 3 meters of distance. Based on these results, we make suggestions for different contexts in which lithics fall into the fire.
\end{abstract}

Keywords: Thermal alteration; Discard; Hearths; Distribution; Experimentation.

Los conjuntos líticos pueden resultar termoalterados por una variedad de situaciones disímiles. Entre ellas podemos incluir prácticas tecnológicas como el tratamiento térmico o el canteo de rocas mediante el fuego; el uso de piedras termóforas como método de cocción o calefacción; la caída de artefactos dentro de estructuras de combustión, por causas accidentales -por ejemplo en contextos en los que la talla lítica se realiza cerca del fuego- o en situaciones intencionales -la incineración de bienes en el marco de ritos funerarios o el descarte de artefactos dentro de fogones -; la acción de fogones encendidos en ocupaciones ocurridas con posterioridad al descarte de los artefactos e incendios naturales. Es decir que a partir del reconocimiento de artefactos líticos termoalterados es posible indagar sobre diversos aspectos de interés arqueológico, como pueden ser procesos postdepositacionales o paleoambientales, cuestiones tecnológicas, prácticas culinarias, rituales o de uso del espacio. En este sentido, la ubicación de restos líticos termoalterados ha sido usada para la reconstrucción de los lugares donde fueron encendidas las fogatas, en aquellos casos en que no quedan evidencias de estructuras de combustión (Cattáneo 2002; Sergant et al. 2006). También puede usarse para evaluar la acción de procesos de formación de sitio (Ortiz Nieto-Márquez \& Baena Preysler 2015).

El estudio de la termoalteración de los artefactos líticos tiene una fuerte base experimental. Los análisis se han centrado principalmente en identificar qué rasgos 
macroscópicos son indicadores del contacto de las rocas con el fuego y en comprender cuales son los cambios estructurales que se producen durante este proceso. Asimismo, como el abordaje ha estado por lo general enfocado en el tratamiento térmico, numerosos estudios han sido realizados para comprender cuales son las consecuencias de este procedimiento pirotecnológico (Domanski \& Webb 2007).

Un aspecto que no ha sido abordado lo suficiente desde los estudios experimentales son las consecuencias en la distribución espacial de los restos termoalterados. Como la localización de los artefactos líticos con respecto al fuego difiere en los diversos procesos de termoalteración y considerando que el tamaño de los artefactos incide en cómo estos responden a la acción térmica (Mandeville 1973), es esperable que se generen diversos patrones distribucionales como consecuencia de la acción del fuego. Es importante destacar que cuando las rocas son expuestas a calentamientos muy rápidos, se fracturan de manera explosiva (Purdy \& Brooks 1971).

En este trabajo, realizamos experimentaciones para evaluar el modo en que se distribuyen los restos líticos arrojados al fuego. El objetivo a nivel general es aportar a los estudios de termoalteración artefactual y, a nivel particular, generar marcos de referencia que nos permitan identificar los procesos de caída de artefactos líticos en contextos arqueológicos de la Meseta Central de Santa Cruz y contribuir así a los análisis de uso del espacio intrasitio llevados adelante en la región. Si bien comprender los procesos de termoalteración requiere el registro de los rasgos generados en los restos, por razones de extensión nos concentraremos en la cuestión espacial.

\section{El estudio de las termoalteraciones en la Meseta Central de Santa Cruz}

El paisaje de la Meseta Central de Santa Cruz muestra gran abundancia y amplia disponibilidad de fuentes de materias primas silíceas de muy buena calidad para la talla. Estas rocas fueron recurrentemente utilizadas por las sociedades que habitaron la región en el pasado. En este marco, desde hace algunos años venimos realizando experimentaciones para evaluar si las sociedades que habitaron este espacio aplicaron el tratamiento térmico de artefactos líticos como parte de sus prácticas de manufactura (Frank 2011). Estas experimentaciones, que complementan y amplían trabajos previos realizados con litologías de la región (Cattáneo et al. 1997-98; Nami et al. 2000), nos han permitido aprender sobre aspectos referidos al modo de llevar adelante este procedimiento y reconocer los indicadores propios de dicha actividad así como aquellos que ocurren cuando el procedimiento falla.

A través de este abordaje hemos podido reconocer, en algunos de los sitios excavados, que el tratamiento térmico fue una técnica aplicada, durante el Pleistoceno final, fundamentalmente para la producción de artefactos bifaciales (Frank 2011). Para este mismo período, identificamos restos líticos de pequeñas dimensiones dañados térmicamente que parecen consistentes con procesos de caída accidental dentro de los fogones durante la formatización final de los instrumentos. Ello es esperable si estas actividades se realizaban cerca de las estructuras de combustión, aspecto que también fue corroborado (Skarbun \& Frank 2011).

Por otra parte, para sitios del Holoceno medio observamos que aumenta en forma considerable la cantidad de restos dañados térmicamente (Cattáneo 2002; Frank 2016). Estos son de mayor tamaño que aquellos dañados durante el Pleistoceno final, incluyen núcleos e instrumentos y fueron hallados por lo general dentro y/o cerca de los fogones. Esto nos ha llevado a considerar la posibilidad de que se haya implementado el descarte intencional de artefactos dentro de estructuras de combustión (Frank 2016). Cabe destacar que a partir del Holoceno medio existiría en el área una mayor intensidad de ocupación, ya sea por un incremento demográfico o por una mayor permanencia de la gente en los sitios (Hermo 2008; Miotti 2006); ello demandaría la implementación de prácticas de mantenimiento del espacio habitado (Frank 2016). El descarte de artefactos grandes dentro de los fogones podría interpretarse dentro del marco de estas prácticas.

Sin embargo, abordar cuestiones vinculadas al uso del espacio intrasitio a partir del reconocimiento de artefactos líticos termoalterados requiere una mejor comprensión de cómo responden las rocas a nivel espacial cuando son expuestas al calor en distintas situaciones. Como mencionamos antes, esto no ha sido abordado sistemáticamente. Los escasos trabajos centrados en analizar el descarte o caída de artefactos en fogones desde una perspectiva experimental en general han puesto el foco en los rasgos de termoalteración generados. En algunos casos se ha evaluado la dispersión de las piezas dentro y fuera del fogón y se ha referido que la distancia máxima desde el fogón a la que se han recuperado restos puede llegar a ser de 2,8 a 3,4 m (Driscoll \& Menuge 2011; Ortiz Nieto-Márquez \& Baena Preysler 2015; Sergant et al. 2006).

\section{Metodología}

Para estudiar cómo se distribuyen los restos líticos descartados en estructuras de combustión realizamos experimentaciones empleando tres materias primas procedentes de la Localidad Arqueológica La María, para las cuales tenemos evidencias de su explotación: sílex rojo, toba silicificada naranja y xilópalo marrón (Fig. 1; para mayor detalle ver Frank 2011). También utilizamos vidrio, el cual sirvió de materia prima para los pueblos 
de la Patagonia a partir del siglo XVI. Para cada materia prima, generamos tres series (de tres lascas cada una) en función de su longitud máxima: Serie A: entre 1 y 2 $\mathrm{cm}$; serie $B$ : entre 3 y $4 \mathrm{~cm}$ y serie $C$ : mayores a $5 \mathrm{~cm}$. Se conformaron así 12 series experimentales, con un total de 36 lascas.

Para cada serie experimental encendimos un fogón, de ca. $50 \mathrm{~cm}$ de diámetro. Controlamos la temperatura empleando una termocupla de tipo K, ubicada directamente debajo de los troncos. Al alcanzar los $500^{\circ}$ $C$ arrojamos las piezas al centro del fogón, manteniendo esta temperatura como mínima durante 30 minutos para luego dejar que el fuego se apague naturalmente. Una vez apagado, recuperamos piezas tanto adentro como afuera del fogón. Para los fragmentos que salieron arrojados fuera del fogón, medimos la distancia en centímetros a partir del borde de la estructura hasta su lugar de caída (Fig. 1). En tanto, en laboratorio registramos las dimensiones de cada fragmento y diversos indicadores de termoalteración.

\section{Resultados}

Una vez arrojadas al fuego, las piezas comienzan a fragmentarse inmediatamente. En aquellos casos en que fragmentos salen despedidos fuera del fogón, esto sucede en los primeros minutos de exposición. Luego se alcanza cierto grado de estabilidad; la fragmentación cesa o disminuye notablemente y los restos se mantienen dentro de la estructura de combustión. En términos generales, los resultados indican que las distintas materias primas responden de manera diversa al calor pero, al mismo tiempo, existen tendencias que están vinculadas al tamaño de los artefactos.

En el caso de la toba silicificada naranja, los fragmentos generados tienden a ser menores a $2 \mathrm{~cm}$ (Tabla 1). El índice de fragmentación es bajo en las series A y B y aumenta considerablemente en la serie de mayor tamaño (C), en la cual se produjeron cerca de 40 fragmentos por cada artefacto arrojado al fuego (Tabla 1). En las series
A y B todos los restos se hallaron dentro de la estructura de combustión. Por el contrario, las piezas que medían más de $5 \mathrm{~cm}$ al fragmentarse expulsaron el 9,2\% de los restos por fuera de la estructura (Tabla 2), llegando a alcanzar los 3,3 metros de distancia. Los fragmentos recuperados fuera de la estructura de combustión en esta última serie miden en su gran mayoría menos de 2 $\mathrm{cm}$ de largo $(90,1 \%)$; sólo dos fragmentos miden entre 2 y 3,9 cm, y fueron recuperados a menos de $20 \mathrm{~cm}$ del borde del fogón.

En el sílex rojo, al igual que con la toba silicificada, la gran mayoría de los fragmentos hallados son menores a $2 \mathrm{~cm}$ (Tabla 1). El índice de fragmentación aumenta considerablemente en la serie $C$, habiéndose producido 393 fragmentos por pieza (Tabla 1). Cabe resaltar que, a igual tamaño, este sílex tiende a fragmentarse muchísimo más que el resto de las materias primas utilizadas. Esta mayor fragilidad de la roca también se expresa en el hecho de que en las series B y C más del 8\% de los restos se hallaron por fuera del fogón (Tabla 2). En la serie de entre 3-4 cm (B) la distancia máxima alcanzada por los fragmentos fue de $85 \mathrm{~cm}$ y todos los restos recuperados fuera del fogón midieron menos de $2 \mathrm{~cm}$ de largo. En tanto, en la serie de más de $5 \mathrm{~cm}$ (C) se llegó a recuperar fragmentos hasta $184 \mathrm{~cm}$ alejados del borde del fogón. En dicha serie el 99,1\% de los fragmentos hallados fuera del fogón miden menos de $2 \mathrm{~cm}$; sólo dos fragmentos miden entre 2 y $3,9 \mathrm{~cm}$, y fueron recuperados a menos de $20 \mathrm{~cm}$ del borde del fogón.

En el caso del xilópalo, en todas las series la mayor proporción de restos mide menos de $2 \mathrm{~cm}$ (Tabla 1). El índice de fragmentación es similar al de la toba silicificada (Tabla 1), aunque resulta interesante que en la serie $A$ ninguna de las piezas se ha fracturado. Mientras que en las series A y B todos los elementos fueron recuperados dentro del fogón, en las piezas que medían más de 5 $\mathrm{cm}$ de longitud un 3\% $(n=4)$ de los fragmentos salieron expulsados (Tabla 2), alcanzando una distancia máxima de $129 \mathrm{~cm}$. Los cuatro restos miden menos de $2 \mathrm{~cm}$ cada uno.

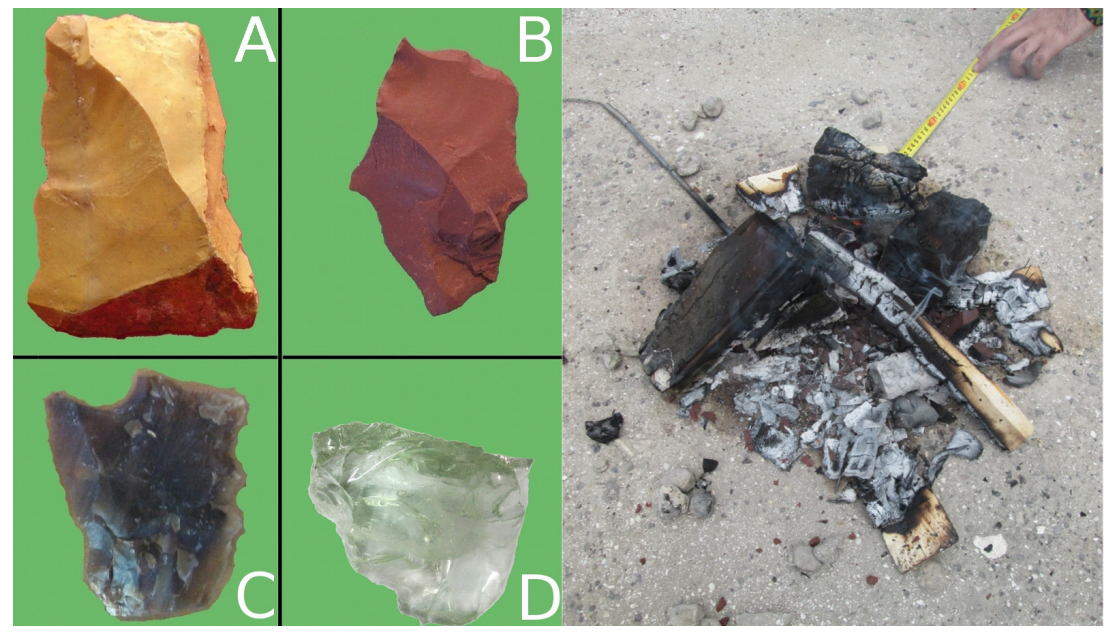

Figura 1. Izq. Materias primas empleadas en la experimentación. A. Toba silicificada. B. Sílex. C. Xilópalo. D. Vidrio. Der. Medición de la distancia de piezas expulsadas del fogón. Nótese en la parte inferior la dispersión de fragmentos de sílex rojo.

Figure 1. Left raw materials used in the experimentation. A. Silicified tuff. B. Flint. C.Silicified wood. D. Glass. Right Distnace measurement of parts ejected from the stove. Note at the bottom the dispersion of red flint fragments. 
Tabla 1. Tamaño de los fragmentos producidos como consecuencia de la termoalteración (n y \%). Índice de fragmentación (n de fragmentos/n de piezas originales).

Table 1. Size of the thermally altered fragments ( $n$ and \%) and fragmentation index in each raw material ( $n$ fragments/n original flakes).

\begin{tabular}{|c|c|c|c|c|c|c|c|c|}
\hline \multirow{2}{*}{$\begin{array}{l}\text { Materia } \\
\text { prima }\end{array}$} & \multirow[b]{2}{*}{ Serie } & \multicolumn{5}{|c|}{ Tamaño fragmentos } & \multirow[b]{2}{*}{ Total } & \multirow{2}{*}{$\begin{array}{l}\text { Índice de } \\
\text { fragmentación }\end{array}$} \\
\hline & & $\begin{array}{c}\text { Menos de } \\
0,5 \mathrm{~cm}\end{array}$ & $\begin{array}{c}0,5 \text { a } 1,9 \\
\mathrm{~cm}\end{array}$ & $\begin{array}{c}2 \text { a } 3,9 \\
\mathrm{~cm}\end{array}$ & $\begin{array}{c}4 \text { a } 5,9 \\
\mathrm{~cm}\end{array}$ & $\begin{array}{c}6 \mathrm{~cm} 0 \\
\text { más }\end{array}$ & & \\
\hline \multirow{3}{*}{$\begin{array}{c}\text { Toba } \\
\text { silicificada }\end{array}$} & A & 0 & $6(100 \%)$ & 0 & 0 & 0 & $6(100 \%)$ & 2 \\
\hline & $B$ & 0 & $5(41,7 \%)$ & $7(58,3 \%)$ & 0 & 0 & $12(100 \%)$ & 4 \\
\hline & C & $25(21 \%)$ & $83(69,7 \%)$ & $9(7,6 \%)$ & $\begin{array}{c}2 \\
(1,7 \%)\end{array}$ & 0 & $119(100 \%)$ & 39,66 \\
\hline \multirow{3}{*}{ Sílex } & A & $3(15,8 \%)$ & $15(78,9 \%)$ & $1(5,3 \%)$ & 0 & 0 & $19(100 \%)$ & 6,33 \\
\hline & B & $33(25,6 \%)$ & $95(73,6 \%)$ & $1(0,8 \%)$ & 0 & 0 & $129(100 \%)$ & 43 \\
\hline & C & $\begin{array}{c}478 \\
(40,5 \%)\end{array}$ & $\begin{array}{c}657 \\
(55,73 \%)\end{array}$ & $41(3,5 \%)$ & $\begin{array}{c}3 \\
(0,25 \%)\end{array}$ & 0 & $\begin{array}{c}1179 \\
(100 \%)\end{array}$ & 393 \\
\hline \multirow{3}{*}{ Xilópalo } & A & 0 & $3(100 \%)$ & 0 & 0 & 0 & $3(100 \%)$ & 1 \\
\hline & B & $3(20 \%)$ & $6(40 \%)$ & $6(40 \%)$ & 0 & 0 & $15(100 \%)$ & 5 \\
\hline & C & $43(32,6 \%)$ & $77(58,3 \%)$ & $12(9,1 \%)$ & 0 & 0 & $132(100 \%)$ & 44 \\
\hline \multirow{3}{*}{ Vidrio } & A & 0 & $4(100 \%)$ & 0 & 0 & 0 & $4(100 \%)$ & 1,33 \\
\hline & B & 0 & 0 & $5(100 \%)$ & 0 & 0 & $5(100 \%)$ & 1,66 \\
\hline & C & 0 & $7(21,2 \%)$ & $\begin{array}{c}16 \\
(48,5 \%)\end{array}$ & $\begin{array}{c}7 \\
(21,2 \%)\end{array}$ & $\begin{array}{c}3 \\
(9,1 \%)\end{array}$ & $33(100 \%)$ & 11 \\
\hline \multicolumn{2}{|c|}{ Total } & $\begin{array}{c}585 \\
(35,3 \%)\end{array}$ & $\begin{array}{c}958 \\
(57,8 \%)\end{array}$ & $98(5,9 \%)$ & $\begin{array}{c}12 \\
(0,7 \%)\end{array}$ & $\begin{array}{c}3 \\
(0,2 \%)\end{array}$ & $\begin{array}{c}1656 \\
(100 \%)\end{array}$ & 46 \\
\hline
\end{tabular}

Tabla 2. Fragmentos recuperados dentro del fogón y a diferentes distancias de él (n y \%).

Table 2. Fragments recovered inside and outside the hearth ( $n$ and \%).

\begin{tabular}{|c|c|c|c|c|c|c|c|c|c|}
\hline Materia prima & $\begin{array}{c}\text { Tamaño } \\
\text { original }\end{array}$ & $\begin{array}{c}\text { Dentro del } \\
\text { fogón }\end{array}$ & $\begin{array}{c}0,1-19 \\
\mathrm{~cm}\end{array}$ & $\begin{array}{c}20- \\
39 \mathrm{~cm}\end{array}$ & $\begin{array}{c}40- \\
59 \mathrm{~cm}\end{array}$ & $\begin{array}{c}60- \\
79 \mathrm{~cm}\end{array}$ & $\begin{array}{c}80-99 \\
\mathrm{~cm}\end{array}$ & $\begin{array}{c}\text { Más de } \\
1 \mathrm{~m}\end{array}$ & $\begin{array}{c}\text { Total } \\
\text { Toba Silicificada }\end{array}$ \\
\hline C & $\begin{array}{c}108 \\
(90,8 \%)\end{array}$ & $\begin{array}{c}3 \\
(2,5 \%)\end{array}$ & $\begin{array}{c}3 \\
(2,5 \%)\end{array}$ & 0 & $\begin{array}{c}1 \\
(0,8 \%)\end{array}$ & 0 & $\begin{array}{c}4 \\
(3,4 \%)\end{array}$ & $\begin{array}{c}119 \\
(100 \%)\end{array}$ \\
\hline Sílex & $\mathrm{B}$ & $\begin{array}{c}118 \\
(91,5 \%)\end{array}$ & $\begin{array}{c}4 \\
(3,1 \%)\end{array}$ & $\begin{array}{c}3 \\
(2,3 \%)\end{array}$ & $\begin{array}{c}2 \\
(1,6 \%)\end{array}$ & $\begin{array}{c}1 \\
(0,8 \%)\end{array}$ & $1(0,8 \%)$ & 0 & $\begin{array}{c}129 \\
(100 \%)\end{array}$ \\
\hline Xilópalo & $\mathrm{C}$ & $\begin{array}{c}1069 \\
(90,7 \%)\end{array}$ & $\begin{array}{c}49 \\
(4,2 \%)\end{array}$ & $\begin{array}{c}27 \\
(2,3 \%)\end{array}$ & $\begin{array}{c}10 \\
(0,8 \%)\end{array}$ & $\begin{array}{c}7 \\
(0,6 \%)\end{array}$ & $4(0,3 \%)$ & $\begin{array}{c}13 \\
(1,1 \%)\end{array}$ & $\begin{array}{c}1179 \\
(100 \%)\end{array}$ \\
\hline
\end{tabular}

Finalmente, el vidrio responde de forma muy diferente a las otras materias primas. La mayoría de los fragmentos son mayores a $2 \mathrm{~cm}$ (Tabla 1). El índice de fragmentación es menor al resto de las materias primas, pero respetando el aumento paulatino a medida que aumenta el tamaño de las lascas originales (Tabla 1). Todos los elementos de cada serie fueron recuperados dentro del fogón.

\section{Discusión y Conclusiones}

En función de estos resultados podemos delinear tendencias y reflexiones que sirven para generar expectativas arqueológicas para este tipo de contextos. Por un lado, vemos que a medida que aumenta el tamaño se incrementa la fragmentación y la dispersión de restos fuera del fogón. Las lascas más grandes al ser arrojadas al fuego tienden a generar muchos restos. Si bien la mayor parte continúa dentro del fogón una vez apagado el fuego, algunos llegan a ser despedidos a largas distancias. Dependiendo de la materia prima, hasta un 9,2\% de los fragmentos pueden ser encontrados fuera del fogón. La mayoría $(41,9 \%)$ de los fragmentos termoalterados recuperados fuera del fogón en las distintas series se hallaron a menos de $20 \mathrm{~cm}$ de distancia de este. Por otra 
parte, las lascas más pequeñas son mucho más resistentes (en algunos casos como el xilópalo, se conservan enteras) y suelen mantenerse dentro de la estructura de combustión.

Al mismo tiempo estas tendencias son dependientes de la materia prima. En este sentido en un extremo está el sílex rojo que tiene alta fragmentación y que expulsa restos fuera del fogón incluso en la serie $B$, mientras que en el otro extremo está el vidrio, con baja fragmentación y manteniéndose todos los fragmentos dentro de la estructura. La toba silicificada y el xilópalo muestran comportamientos intermedios.

Con respecto al tamaño de los fragmentos generados, para las rocas criptocristalinas suelen prevalecer restos menores a dos $\mathrm{cm}$, mientras que en el vidrio los fragmentos predominantes miden entre 3 y $4 \mathrm{~cm}$ de longitud máxima. Un rasgo relevante es que los fragmentos termoalterados de mayor tamaño tienden a mantenerse dentro del fogón. En muy pocas ocasiones se recuperaron fragmentos de $2 \mathrm{~cm}$ o más de longitud por fuera del fogón (series C de toba silicificada y sílex), y en dichos casos siempre se localizaron a menos de $20 \mathrm{~cm}$ de distancia del borde de la estructura. Por el contrario, los fragmentos menores pueden salir desprendidos a grandes distancias llegando a superar el metro de distancia y alcanzar inclusive los $330 \mathrm{~cm}$.

Estas observaciones nos permiten generar una serie de expectativas para distintos contextos de caída de piezas al fuego. En los sitios estudiados de la Meseta Central predominan las prácticas de formatización final de artefactos alrededor de los fogones. En consecuencia, se espera que caigan accidentalmente esquirlas y microesquirlas dentro de las estructuras de combustión. En dichos casos, los restos tenderían a mantenerse dentro de la estructura, presentarían un bajo nivel de fractura, y estarían asociados contextualmente a otros productos de talla de la misma materia prima (no termoalterados) ubicados alrededor del fogón. Por otra parte, en situaciones de descarte intencional de artefactos (ya sea en el marco de prácticas rituales o por el mantenimiento de los espacios) suelen arrojarse al fuego mayor diversidad de grupos tipológicos incluyendo instrumentos y núcleos de gran tamaño. En esas ocasiones esperaríamos una mayor cantidad de fragmentos. Estos serían predominantemente menores a dos $\mathrm{cm}$ pero también se deberían hallar dentro de la estructura elementos de mayor tamaño. Dependiendo de la materia prima, es factible que algunos de los fragmentos se hallen dispersos fuera del fogón, por lo general concentrados en los primeros $20 \mathrm{~cm}$ alrededor de la estructura pero también, en baja proporción podrían hallarse a grandes distancias. Estos restos hallados fuera del fogón deberían ser, fundamentalmente, menores a dos cm de longitud máxima. Asimismo, en este tipo de prácticas no se espera que exista necesariamente asociación contextual con otros restos de la misma materia prima.

Consideramos entonces, y para finalizar, que la correcta identificación de restos líticos termoalterados y su localización precisa en el espacio dentro de los sitios brinda rica información para interpretar diversas prácticas humanas y procesos naturales. Procuraremos continuar con las experimentaciones para aportar a la mejor comprensión de dichos procesos.

La Plata, 15 de febrero de 2019

\section{Bibliografía}

Cattáneo, G. R. (2002). Una aproximación a la organización de la tecnología lítica entre los cazadores recolectores del Holoceno Medio/Pleistoceno Final en la Patagonia Austral, Argentina. Tesis doctoral Inédita, Universidad Nacional de La Plata, La Plata.

Cattáneo, G. R., Pupio, A., Valente, M., \& Barna, A. (1997-98). Alteración térmica en dos tipos de rocas silíceas: resultados experimentales y aporte de datos para el análisis arqueológico. Relaciones de la Sociedad Argentina de Antropología, XXII-XXIII, 343-361.

Domanski, M., \& Webb, J. (2007). A Review of Heat Treatment Research. Lithic Technology, 32 (2), 153 - 194.

Driscoll, K., \& Menuge, J. (2011). Recognising burnt vein quartz artefacts in archaeological assemblages. Journal of Archaeological Science, 38, 2251-2260.

Frank, A. D. (2011). Tratamiento térmico y manejo del fuego en sociedades cazadoras-recolectoras de la Meseta Central de Santa Cruz. Tesis doctoral Inédita, Universidad Nacional de La Plata, La Plata.

Frank, A. D. (2016). Tratamiento térmico de artefactos líticos en Cerro Tres Tetas 1, meseta central de Santa Cruz, Patagonia Argentina. En F. Mena (Ed.), Arqueología de la Patagonia: de Mar a Mar (pp. 367-378). Coyhaique: Ediciones CIEP y Ñire Negro.

Hermo, D. (2008). Los cambios en la circulación de las materias primas líticas en ambientes mesetarios de Patagonia. Una aproximación para la construcción de los paisajes arqueológicos de las sociedades cazadorasrecolectoras. Tesis doctoral Inédita, Universidad Nacional de La Plata, La Plata.

Mandeville, M. (1973). A consideration of the thermal pretreatment of chert. Plains Anthropologist, 18, 177 - 202.

Miotti, L. L. (2006). Paisajes domésticos y sagrados desde la arqueología de los cazadores-recolectores en el Macizo del Deseado, Provincia de Santa Cruz. Cazadores Recolectores del Cono Sur. Revista de Arqueología, 1, 11-40. 
Nami, H. G., Cattáneo, G. R., \& Pupio, M. (2000). Investigaciones experimentales sobre el tratamiento térmico en algunas materias primas de Pampa y Patagonia. Anales del Instituto de la Patagonia (Serie Ciencias Sociales), 28, 315-329.

Ortiz Nieto-Márquez, I., \& Baena Preysler, J. (2015). Experiments around the fire. Discovering human and natural processes in Middle Palaeolithic hearths. Anthropologie. International Journal of Human Diversity and Evolution, LIII(3), 501-518.

Purdy, B., \& Brooks, H. (1971). Thermal alteration of silica minerals: an archaeological approach. Science, $173,322-325$.

Sergant, J., Crombe, P., \& Perdaen, Y. (2006). The 'invisible' hearths: a contribution to the discernment of Mesolithic non-structured surface hearths. Journal of Archaeological Science, 33, 999 - 1007.

Skarbun, F., \& Frank, A. D. (2011). Organización espacial intrasitio durante el Pleistoceno final en la Meseta Central de Santa Cruz. Evidencias del sitio Casa del Minero 1. Relaciones de la Sociedad Argentina de Antropología, XXXVI, 289-313. 